\title{
Coupled identical localized fermionic chains with quasirandom disorder
}

\author{
Vieri Mastropietro \\ Universitá di Milano, Via C. Saldini 50, 20133 Milano, Italy \\ (Received 2 January 2017; published 28 February 2017)
}

\begin{abstract}
We analyze the ground-state localization properties of an array of identical interacting spinless fermionic chains with quasirandom disorder, using nonperturbative renormalization-group methods. In the single- or two-chain case localization persists, while for a larger number of chains a different qualitative behavior is generically expected, unless the many-body interaction is vanishing. This is due to number-theoretical properties of the frequency, similar to the ones assumed in Kolmogorov-Arnold-Moser theory, and cancellations due to the Pauli principle, which in the single- or two-chain case imply that all the effective interactions are irrelevant; in contrast, for a larger number of chains, relevant effective interactions are present.
\end{abstract}

DOI: 10.1103/PhysRevB.95.075155

\section{INTRODUCTION}

A quantum system in which disorder-induced localization [1] persists in the presence of an interaction is said to be in a many-body localized phase. While normal systems are expected asymptotically to approach a thermal state (due to the interaction, "the system acts as its own bath"), this does not happen in a many-body localized phase [2-4], a fact with deep theoretical and technological implications. However, the interplay of disorder and interaction produces a complex behavior [5,6], and the existence itself of a many-body localized phase is quite a nontrivial property that is under thorough investigation.

In the case of random disorder, many-body localization (MLB) was established order by order by a formal series in any dimension [7-9], but this does not exclude delocalization due to the possible divergence of the expansions. In one dimension, a nonperturbative proof of MBL has been reached [10,11], but it relies on a still unproven assumption. Numerical evidence of MBL in one-dimensional lattices was obtained in [12-14].

In addition, quasirandom disorder in one dimension produces localization in the single-particle case, as found in [15] and rigorously proved in $[16,17]$. In the presence of interaction, a nonperturbative proof of ground-state localization was achieved in [18]. Numerical evidence of MBL with quasirandom disorder was found in [19-23]. One-dimensional systems of particles with quasirandom disorder can be realized in cold-atoms experiments [24], and evidence of MBL was claimed.

As a natural step toward higher dimensions, we consider an array of interacting fermionic chains with Aubry-André quasirandom disorder [15] and coupled by a hopping term. Such a model (with spinful fermions) has been realized in cold-atom experiments in [25]. We call $x=0, \pm 1, \pm 2, \ldots$ the coordinates of the infinite chain, and $y=0, \ldots, L$ are the coordinates labeling the chains, and we consider a system of $N$ spinless fermions with Hamiltonian

$$
H_{N}=\sum_{i=1}^{N} H_{A}\left(x_{i}\right)+J_{\perp} \sum_{i=1}^{N} \nabla_{y_{i}}+U \sum_{i, j \in 1}^{N} v\left(x_{i}-x_{j}\right),
$$

where $v(x-y)=\delta_{y, x+1}, H_{A}$ is the Aubry-André Hamiltonian,

$$
H_{A}(x)=J \nabla_{x}+\Delta \cos [2 \pi(\omega x+\theta)],
$$

and $\nabla_{z} f(z)=f(z-1)+f(z+1)-2 f(z)$; periodic boundary conditions are imposed in $y$. The Hamiltonian (1) describes $L$ fermionic chains, with identical disorder, intrachain hopping $J$, intrachain interaction $U$, and interchain hopping $J_{\perp}$. If $J_{\perp}=U=0$, the system reduces to several uncoupled AubryAndré models [15]. The behavior of the eigenfunctions of $H_{A}(2)$ depends crucially on the ratio $\frac{\Delta}{J}$ between the disorder and the hopping; if $\frac{\Delta}{J}<2$, the eigenfunctions are quasi-Bloch extended waves, while for $\frac{\Delta}{J}>2$ the eigenfunctions are exponentially decaying, and Anderson localization occurs [16,17]. A metal-insulator transition is therefore present varying the strength of the disorder, a feature making quasirandom disorder somewhat similar to random disorder in three dimensions.

The question we address in this paper is whether a localized phase persists in the array described by (1), and how the behavior depends on the interplay between the hopping $J_{\perp}$, the interaction $U$, and the number of chains $L$. The main theoretical difficulty is that localization is a nonperturbative phenomenon; the presence or absence of localization is related to the convergence or divergence of the series, driven by small divisors that can produce dangerous factorials. Information is carried by high orders, and instability is not signaled by divergences at low orders, as happens in quantum field theory.

In the single-particle case $H_{A}$, the small divisors are similar to the ones in the series in Kolmogorov-ArnoldMoser (KAM) theory, whose convergence implies stability in close-to-integrable system, while divergence is related to the onset of chaos. Indeed, the eigenfunctions of (2) can be written in a series of $J$, and divisors are of the form $\phi_{x}-\phi_{y}$ with $x \neq y$, with $\phi_{x}=\Delta \cos [2 \pi(\omega x+\theta)]$. To get convergence, and as a consequence localization, one needs to assume number-theoretical conditions, called Diophantine (see below), to control the size of $\|(\omega n)\|$ and $\|(\omega n+2 \theta)\|$, with $\|\cdot\|$ the norm on the side 1 torus; see $[16,17]$. Such Diophantine conditions are the same as those assumed in KAM theory. In the presence of interaction, the small divisors in the expansion for the $N$-particle eigenfunctions are much more complex; they are of the form $E_{N}(\underline{x})-E_{N}(y)$, with $E_{N}(\underline{x})=\sum_{i=1}^{N} \phi_{x_{i}}$. No number-theoretical condition is known to control them for $N>1$ [26] (for the $N=2$ case, see [27]).

Even if the construction of all the eigenfunctions of (1) for a generic $N$ is outside the present analytical possibilities, we can analyze the problem using a different approach, 
introduced in [18]: we do not consider the expansion for the eigenfunctions, but we compute in the thermodynamic limit $N \rightarrow \infty$ the grand-canonical correlations, which at zero temperature become the ground-state correlations. This approach allows us to take advantage of fermionic cancellations, and nonperturbative and rigorous information on the localization of systems with an infinite number of particles, even if limited to the ground state, can be obtained. The correlations are written as Grassmann integrals that are analyzed via exact fermionic renormalization-group $(\mathrm{RG})$ methods; one integrates out the degrees of freedom with smaller and smaller energy obtaining a sequence of effective interactions, given by a sum of terms that are all relevant in the RG sense, independently of the number of fields. The presence of an infinite number of relevant processes seems to say that an RG approach is hopeless; however, by exploiting number-theoretical properties of the frequency of incommensurate disorder, it is possible to show that a huge class of effective interactions, called nonresonant, is indeed irrelevant. In contrast with the single-chain problem, in which Diophantine conditions are sufficient, here one needs other conditions as well, called in KAM theory the first and second Melnikov conditions. While in the absence of interaction the structure of Feynman graphs is rather simple, the presence of interaction $U \neq 0$ complicates the problem considerably; one has a combination of small divisors and loops, which are absent in noninteracting or KAM-like problems. Other dangerous factorials, in addition to the ones produced by small divisors, are produced by combinatorics related to the number of graphs; they are controlled by cancellations due to the fermionic sign cancellations.

A renormalized expansion is obtained in terms of the running coupling constants corresponding to the resonant terms. As usual in a RG, the physical properties depend on their flow; if the running coupling constants do not exit from the convergence radius, the interacting theory is analytically close to the free one, so that localization persists in the presence of interaction. The flow depends dramatically on the number of chains. In the two-chain problem, there are no relevant effective quartic interactions, the only relevant couplings being quadratic, as in the single-chain problem; localization persists in the ground state in the presence of interaction.

On the contrary, with a higher number of chains the quartic terms are relevant, and their size increase iterates the $\mathrm{RG}$; therefore, a different qualitative behavior is generically expected, unless the many-body interaction is vanishing, where localization still persists.

The content of this paper is the following. In Sec. II we present the main results. In Sec. III we perform an exact RG analysis, and we show the irrelevance of the nonresonant terms. In Sec. IV we identify the relevant and marginal terms and study the corresponding flow, and in Sec. V we get our main results discussing the convergence of the expansion. Finally, in Sec. VI the main conclusions are presented.

\section{MAIN RESULT}

We consider the grand-canonical averages $\langle O\rangle=$ $\sum_{N} \frac{\operatorname{Tr}_{N} e^{-\beta\left(H_{N}-\mu N\right)} O}{Z}$, with $Z=\sum_{N} \operatorname{Tr}_{N} e^{-\beta\left(H_{N}-\mu N\right)}$; the thermodynamic limit is taken sending the chain length to infinity keeping the number of chains $L$ finite. The Fock space
Hamiltonian is

$$
\begin{aligned}
H= & J \sum_{x, y}\left(a_{x+1, y}^{+} a_{x, y}^{-}+a_{x-1, y}^{+} a_{x, y}^{-}\right) \\
& +\Delta \sum_{x, y} \cos (2 \pi \omega x) a_{x, y}^{+} a_{x, y}^{-} \\
& +U \sum_{x, y} a_{x, y}^{+} a_{x, y}^{-} a_{x+1, y}^{+} a_{x+1, y}^{-} \\
& +J_{\perp} \sum_{x, y}\left(a_{x, y+1}^{+} a_{x, y}^{-}+a_{x, y}^{+} a_{x, y+1}^{-}\right),
\end{aligned}
$$

and we assume for definiteness the phase of the disorder equal to zero. It is convenient to write $a_{x, y}^{ \pm}=\frac{1}{L} \sum_{l} e^{ \pm i l y} \widehat{a}_{x, l}^{ \pm}$, where $l=2 \pi \frac{n}{L}$, with $n=0, \ldots, L-1$, so that the Hamiltonian can be rewritten in the following way:

$$
\begin{aligned}
H= & J \frac{1}{L} \sum_{x, l}\left(\widehat{a}_{x+1, l}^{+} \widehat{a}_{x, l}^{-}+\widehat{a}_{x-1, l}^{+} \widehat{a}_{x, l}^{-}\right) \\
& +\frac{\Delta}{L} \sum_{x, l} \cos (2 \pi \omega x) \widehat{a}_{x, l}^{+} \widehat{a}_{x, l}^{-} \\
& +\frac{J_{\perp}}{L} \sum_{x, l}(2 \cos l) \widehat{a}_{x, l}^{+} \widehat{a}_{x, l}^{-}+U \sum_{x} \frac{1}{L^{4}} \\
& \times \sum_{l_{1}, l_{2}, l_{3}, l_{4}} \widehat{a}_{x, l_{1}}^{+} \widehat{a}_{x, l_{2}} \widehat{a}_{x+1, l_{3}}^{+} \widehat{a}_{x+1, l_{4}}^{-} \delta\left(l_{1}-l_{2}+l_{3}-l_{4}\right) .
\end{aligned}
$$

We focus on the two-point function $\left\langle\widehat{a}_{\mathbf{x}, l}^{-} \widehat{a}_{\mathbf{z}, l}^{+}\right\rangle$, where $\langle O\rangle=\frac{\operatorname{Tr} e^{-\beta(H-\mu N)} T O}{\operatorname{Tr} e^{-\beta(H-\mu N)}}, T$ is the time ordering, and $\widehat{a}_{\mathbf{x}, l}^{ \pm}=$ $e^{(H-\mu N) x_{0}} \widehat{a}_{x, l}^{ \pm} e^{-(H-\mu N) x_{0}}$ and $\mathbf{x}=\left(x_{0}, x\right)$. In the molecular limit $U=J=0$, one has (setting $\Delta=1$ for definiteness)

$$
H_{0}-\mu N=\frac{1}{L} \sum_{x, l}\left[\cos (2 \pi \omega x)-\mu_{l}\right] \widehat{a}_{x, l}^{+} \widehat{a}_{x, l}^{-}
$$

with

$$
\mu_{l}=\mu-2 J_{\perp} \cos l \equiv \cos 2 \pi \omega \bar{x}_{l}
$$

so that, calling $\mu=\cos (2 \pi \omega \bar{x})$, then $\bar{x}_{l}=\bar{x}+a \cos l J_{\perp}$ with $a^{-1}=\pi \omega \sin 2 \pi \omega \bar{x}+O\left(J_{\perp}\right)$. In this limit, the system is uncoupled with an $l$-dependent chemical potential for any chain. The ground-state occupation number is $=1$ for $\cos (2 \pi \omega x)<\mu_{l}$ and 0 for $\cos (2 \pi \omega x)>\mu_{l}$. The two-point function $\left.\left\langle\widehat{a}_{\mathbf{x}, l} \widehat{a}_{\mathbf{y}, l}^{+}\right\rangle\right|_{U=J=0} \equiv g_{l}(\mathbf{x}, \mathbf{y})$ is equal to

$$
g_{l}(\mathbf{x}, \mathbf{y})=\delta_{x, y} \frac{1}{\beta} \sum_{k_{0}=\frac{2 \pi}{\beta}\left(n_{0}+\frac{1}{2}\right)} \widehat{g}_{l}\left(x, k_{0}\right) e^{-i k_{0}\left(x_{0}-y_{0}\right)}
$$

with

$$
\begin{aligned}
\widehat{g}_{l}\left(x, k_{0}\right) & =\int_{0}^{\beta} d \tau e^{i \tau k_{0}} \frac{e^{-\tau\left(\cos 2 \pi \omega x-\mu_{l}\right)}}{1+e^{-\beta\left(\cos 2 \pi \omega x-\mu_{l}\right)}} \\
& =\frac{1}{-i k_{0}+\cos 2 \pi \omega x-\cos 2 \pi \omega \bar{x}_{l}} .
\end{aligned}
$$

The two-point function is perfectly localized in the chain direction (the two-point function is vanishing if $x \neq y$ ), but not in the transversal direction in the coordinate space. Assume that $\bar{x}_{l}$ is not a point of the lattice, so that the propagator (8) 
is never singular. As $\omega$ is an irrational number, $\omega x$ modulo 1 fills densely the set $[-1 / 2,1 / 2)$, and in particular it can be arbitrarily close to $\pm \omega \bar{x}_{l}$. If we set $x=x^{\prime}+\rho_{l} \bar{x}_{l}$, then for small $\left(\omega x^{\prime}\right)_{\bmod 1}, \rho_{l}= \pm$,

$$
\widehat{g}_{l}\left(x, k_{0}\right) \sim \frac{1}{-i k_{0}+v_{l} \rho_{l}\left(\omega x^{\prime}\right)_{\bmod 1}},
$$

and $v_{l}=-2 \pi \omega \sin 2 \pi \omega \bar{x}_{l}$. The expansion of the two-point function in terms of $J, U$ can be represented in terms of Feynman graphs, expressed by the product of propagators $\widehat{g}_{l}\left(x, k_{0}\right)$; on each line of the diagram is associated a coordinate $x$, and the difference of lines coming in or out from the vertex $J$ is \pm 1 , while from a $U$ vertex it is $0, \pm 1$. Note the similarity of (8) with the two-point function in the free fermion limit $\Delta=U=J_{\perp}=0, J=1$, which in Fourier space is given by $1 /-i k_{0}+\cos k-\mu$. If $k=k^{\prime} \pm p_{F}, \mu=$ $\cos p_{F}$, the free fermion propagator is asymptotically given by $1 /-i k_{0} \pm v_{F} k^{\prime}$, which is the well-known Luttinger liquid propagator. $p_{F}$ are called Fermi momenta, and by analogy we can call $\pm \bar{x}_{l}$ the Fermi coordinates.

The expansion in $J, U$ around the molecular limit is convergent at finite temperature, as the temperature acts as an infrared cutoff, and the main issue is to get the zero-temperature limit. We expect that the interaction produces a renormalization of the chemical potential, and it is convenient to fix the renormalized chemical potential to a $J, U$-independent value; this corresponds to fixing the density of the interacting system. We therefore write

$$
\mu_{l}=\cos 2 \pi \omega \bar{x}_{l}+v_{l},
$$

where $v_{l}$ is a counterterm to be fixed so that the chemical potential of the interacting theory is $\cos 2 \pi \omega \bar{x}_{l}$. To understand the behavior at high orders, one needs to exploit some numbertheoretical property of $\omega$; in particular, as in the analysis of the Aubry-André model, we assume that the frequency $\omega$ is a Diophantine number, verifying the property

$$
\|\omega x\| \geqslant C_{0}|x|^{-\tau} \quad \forall x \in \mathbb{Z} /\{0\},
$$

where $\|\cdot\|$ is the norm on the one-dimensional torus. Such a property, saying roughly speaking that $\omega$ is a "good" irrational, is not restrictive as Diophantine numbers have full measure. As an example, the golden ratio $\omega=\frac{\sqrt{5}+1}{2}$ verifies (11) with $\tau=1$ and $C_{0}=\frac{3+\sqrt{5}}{2}$. The Diophantine condition will ensure that a process involving fermions living close to $\left(\omega \bar{x}_{l}\right)$ involves a huge difference of coordinates.

In addition, one has to assume a diophantine condition on the chemical potential (equivalently one can assume a similar condition on $\theta$ ), namely

$$
\|\omega x \pm 2 \omega \bar{x}\| \geqslant C_{0}|x|^{-\tau} \quad \forall x \in \mathbb{Z} /\{0\},
$$

where $\|\cdot\|$ is the norm on the one-dimensional torus. In the decoupled case $J_{\perp}=0$, this implies that $\left|\widehat{g}_{l}\left(x, k_{0}\right)\right| \leqslant C|x|^{\tau}$. Our main result is the following.

If $U, J_{\perp}, J$ are small, $J_{\perp} \neq 0$ belongs to a set of large relative measure, and if $\omega, \bar{x}$ verify (11) and (12), for suitable $\nu_{l}$, then we have the following: (a) If $L=2$ for $\beta \rightarrow \infty$, then for any integer $N$ and a suitable constant $C_{N}$,

$$
\left|\left\langle\widehat{a}_{\mathbf{x}, l}^{-} \widehat{a}_{\mathbf{y}, l}^{+}\right\rangle\right| \leqslant e^{-\xi|x-y|} \frac{C_{N}|\log \Delta|}{1+\left(\Delta\left|x_{0}-y_{0}\right|\right)^{N}}
$$

with $\quad \xi=|\log \varepsilon|, \quad \varepsilon=\max (|J|,|U|), \quad$ and $\quad \Delta=$ $[1+\min (|x|,|y|)]^{-\tau}$.

(b) If $L \geqslant 3, U=0$, then for $\beta \rightarrow \infty$ (13) holds.

(c) If $L \geqslant 3$, then (13) holds for $\beta|U| \leqslant 1$, with $\xi=$ $\max \left(|\log \varepsilon|, \beta^{-1}\right)$ and $\Delta=\max \left((1+\min (|x|,|y|))^{-\tau}, \beta^{-1}\right)$.

In the case of two chains [case (a)], the two-point function decays at zero temperature exponentially in the direction of the chains, and a very weak decay is present in the imaginary time direction faster than any power but with a rate which can be arbitrarely small; this is very similar to what happens in the single-chain case, and it indicates localization of the ground state with or without interaction. In contrast, for a greater number of chains, the interaction produces a qualitative difference; in the absence of many-body interaction, zero-temperature exponential decay is found for any number of chains [case (b)], while in the presence of interaction, convergence of the expansion holds only up to a finite temperature [case (c)]. The reason is that when $L \geqslant 3$, there are extra relevant terms which increase under the RG iterations, and this has the effect that convergence holds only for temperatures not too small. Indeed when $L=2$, the Fermi coordinates are different at different $l$, so that there are no relevant processes connecting them, in contrast to what happens when $L \geqslant 3$. As the presence of diverging directions in the RG flow signals an instability that can imply delocalization, this is in agreement with the behavior observed in cold-atom experiments [25], in which the absence of localization is found in an array of chains (except when there is no interaction, when localization is found), and localization is found in the single-chain case; moreover, we find localization with two chains in the spinless case, a prediction that is accessible in principle to future experiments.

\section{RENORMALIZATION-GROUP ANALYSIS}

The two-point function is obtained by the second derivative of the generating function,

$$
e^{W(\phi)}=\int P(d \psi) e^{V(\psi)+(\psi, \phi)},
$$

with

$$
\begin{aligned}
V= & \frac{1}{L} \sum_{l} \int d \mathbf{x} J\left(\psi_{\mathbf{x}, l}^{+} \psi_{\mathbf{x}+\mathbf{e}_{1}, l}^{-}+\psi_{\mathbf{x}+\mathbf{e}_{\mathbf{1}}, l}^{+} \psi_{\mathbf{x}, l}^{-}\right) \\
& +\int d \mathbf{x} \frac{U}{L^{4}} \sum_{\underline{l}} \psi_{\mathbf{x}, l_{1}}^{+} \psi_{\mathbf{x}, l_{2}}^{-} \psi_{\mathbf{x}+\mathbf{e}_{1}, l_{3}}^{+} \psi_{\mathbf{x}+\mathbf{e}_{1}, l_{4}}^{-} \delta\left(l_{1}-l_{2}+l_{3}-l_{4}\right) \\
& +\frac{1}{L} \sum_{l} v_{l} \int d \mathbf{x} \psi_{\mathbf{x}, l}^{+} \psi_{\mathbf{x}, l}^{-},
\end{aligned}
$$

where $\psi$ are Grassmann variables, $\phi$ is the external source, $\int d \mathbf{x}=\int d x_{0} \sum_{x}, \mathbf{e}_{1}=(0,1)$, and $P(d \psi)$ is the fermionic integration with propagator (7).

We introduce a cutoff smooth function $\chi_{\rho}\left(k_{0}, x\right)$ which is nonvanishing for $\sqrt{k_{0}^{2}+\left\{v_{l}\left[\omega\left(x-\rho \bar{x}_{l}\right)_{\text {mod.1 }}\right]^{2}\right\}} \leqslant \gamma$, where 
$\rho= \pm 1$, and $\gamma>1$ is a suitable constant (to be fixed below); therefore, we can write the propagator as

$$
\widehat{g}_{l}(\mathbf{x})=\widehat{g}_{l}^{(u \cdot v \cdot)}(\mathbf{k})+\sum_{\rho= \pm} \widehat{g}_{\rho, l}(\mathbf{k}),
$$

where

$$
\widehat{g}_{\rho, l}\left(k_{0}, x\right)=\frac{\chi_{\rho}\left(k_{0}, x\right)}{-i k_{0}+\cos [2 \pi(\omega x)]-\cos \left[2 \pi\left(\omega \bar{x}_{l}\right)\right]}
$$

and correspondingly $\psi_{k_{0}, x, l}=\psi_{k_{0}, x, l}^{(u . v .)}+\sum_{\rho= \pm 1} \psi_{\rho ; k_{0}, x, l}$. This simply says that we are rewriting the fermionic field as a sum of two independent fields living close to one of the Fermi points, up to a regular field. We can further decompose

$$
\widehat{g}_{\rho, l}\left(k_{0}, x\right)=\sum_{h=h_{\beta}}^{0} \widehat{g}_{\rho, l}^{(h)}\left(k_{0}, x\right)
$$

with $-h_{\beta} \sim \log \beta, \widehat{g}_{\rho, l}^{(h)}\left(k_{0}, x\right)$ similar to $\widehat{g}_{\rho, l}\left(k_{0}, x\right)$ with $\chi$ replaced by $f_{h}$ with $f_{h}\left(k_{0}, \omega x^{\prime}\right)$ nonvanishing in a region $\sqrt{k_{0}^{2}+\left[v_{l}\left(\omega x^{\prime}\right)_{\bmod 1}\right]^{2}} \sim \gamma^{h}$ with $x=x^{\prime}+\rho \bar{x}_{l}$.

After the integration of the fields, $\psi^{(u \cdot v .)}, \psi^{(0)}, \ldots, \psi^{(h+1)}$, the generating function has the form

$$
e^{W(\phi)}=\int P\left(d \psi^{\leqslant h}\right) e^{V^{(h)}(\psi)+B^{(h)}(\psi, \phi)},
$$

where $P\left(d \psi^{\leqslant h}\right)$ has propagator $g_{\rho, l}^{(\leqslant h)}=\sum_{k=-\infty}^{h} g_{\rho, l}^{(k)}$, and $V^{(h)}(\psi)$ is given by a sum of terms,

$$
\begin{aligned}
& \sum_{x_{1}^{\prime}} \int d x_{0,1} \cdots \int d x_{0, m} \frac{1}{L^{m}} \sum_{l_{1}, \ldots, l_{m}} W_{m, \underline{l}}^{(h)}\left(x_{1}^{\prime}, \underline{x}\right) \\
& \quad \times \delta\left(\sum_{i} \varepsilon_{i} l_{i}\right) \psi_{\rho_{1} ; x_{0,1}, x_{1}^{\prime}, l_{1}}^{\varepsilon_{1}(\leqslant h)} \cdots \psi_{\rho_{m} ; x_{0, m}, x_{m}^{\prime}, l_{m}}^{\varepsilon_{m}(\leqslant h)},
\end{aligned}
$$

where Kronecker $\delta$ 's in the propagators imply that a single sum over $x$ is present; the kernels $W_{m}^{(h)}$ are a sum of Feynman diagrams obtained connecting vertices $J, U$, or $v$ with propagators $g^{(k)}$ with $k>h$. Similarly, $B^{(h)}$ is given by a similar expression, with the only difference being that some of the external lines are associated with $\phi$ fields. The scaling dimension of the theory can be obtained by the bounds

$$
\int d x_{0}\left|g_{\rho}^{(h)}\left(x_{0}, x\right)\right| \leqslant C \gamma^{-h}, \quad\left|g_{\rho}^{(h)}\left(x_{0}, x\right)\right| \leqslant C .
$$

The persistence (or lack thereof) of localization is related to the presence or lack of convergence, that is, to the behavior at high orders; therefore, we need to remember some basic tools of renormalization theory, which are crucial to avoid the well-known problem of "overlapping divergences." Given a Feynman graph, one considers a maximally connected subset of lines corresponding to propagators with scale $h \geqslant h_{v}$ with at least a scale $h_{v}$, and we call it cluster $v$; see Fig. 1 (for more details, see [28]). The external lines have a scale smaller than $h_{v}$. Therefore, to each Feynman graph is associated a hierarchy of clusters; inside each cluster $v$ there are $S_{v}$ maximal clusters, that is, clusters contained only in the cluster $v$ and not in any smaller one, or trivial clusters given by a single vertex. The clusters, therefore, identify the subdiagrams that one needs to

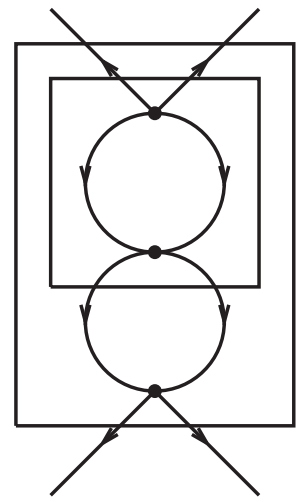

FIG. 1. A graphs and the corresponding clusters, with scales $h_{v_{1}}$ (the smaller) and $h_{v_{2}}$ (the larger), $h_{v_{1}}>h_{v_{2}}, S_{v_{1}}=S_{v_{2}}=2$; the bound (28) is in this case simply $C^{2} \varepsilon^{2} \gamma^{-h_{v_{1}}} \gamma^{-h_{v_{2}}}$.

renormalize as those containing propagators living at energy scales greater than the ones outside them.

By using a tree of propagators with scale $h_{v}$, by integrating the propagators over time, and by using (21) we get that each graph of order $n$ contributing to $W_{m}^{(h)}$ is bounded at fixed scale, if $\varepsilon=\max (|J|,|U|)$, by

$$
C^{n} \varepsilon^{n} \prod_{v} \gamma^{-h_{v}\left(S_{v}-1\right)},
$$

where $v$ are the clusters (not end points) and $h_{v} \leqslant 0$. From the above estimate, we see that the scaling dimension of any contribution to the effective potential has the same positive scaling dimension (independent of the number of fields),

$$
D=1 \text {. }
$$

In other words, all the effective interactions are relevant in the $\mathrm{RG}$ sense, and the theory is nonrenormalizable. Indeed, graphs with all the assignments of scales contribute to the effective potential, and from (22) the sum over scales gives an infinite result. However, it turns out, as a consequence of numbertheoretical properties of the quasirandom disorder, that a huge class of terms is indeed irrelevant. In a large relative measure set of $J_{\perp}$, one has

$$
\left\|\omega x \pm 2 \omega \bar{x}_{l}\right\| \geqslant C_{0}|x|^{-\tau^{\prime}} \quad \forall x \in \mathbb{Z} /\{0\}
$$

and

$$
\left\|\omega x \pm \omega \bar{x}_{l} \pm \omega \bar{x}_{l^{\prime}}\right\| \geqslant C_{0}|x|^{-\tau^{\prime}} \quad \forall x \in \mathbb{Z} /\{0\} .
$$

Conditions (24) and (25) are known in KAM theory as the first and second Melnikov conditions. The first condition is used to bound the propagator; using $\left\|\omega x^{\prime}\right\|=\left\|\omega x-\rho \omega \bar{x}_{l}\right\|=$ $\frac{1}{2}|| \omega 2 x-2 \rho \omega \bar{x}_{l}||$ for small ||$\omega x^{\prime} \|$, then $\left|\widehat{g}^{(h)}\left(k_{0}, x\right)\right| \leqslant C|x|^{\tau}$. The second condition is used to show the irrelevance of a number of terms in the effective potential. Let us consider a contribution to the effective potential (20) with external lines $\psi_{\rho_{1} ; x_{0,1}, x_{1}^{\prime}, l_{1}}^{\varepsilon_{1}(\leqslant h)} \cdots \psi_{\rho_{m} ; x_{0, m}, x_{m}^{\prime}, l_{m}}^{\varepsilon_{m}(\leqslant h)}$. By construction, the coordinates of the external fields are such that $\left(\omega x^{\prime}\right)_{\bmod 1} \leqslant \gamma^{h}$. Note that in each graph there is a tree of propagators connecting all the vertices and external lines (see Fig. 2); each propagator carries a coordinate $x$, and vertices connect lines with coordinates differing at most by \pm 1 ; more exactly, if $x_{i}, x_{j}$ are the 


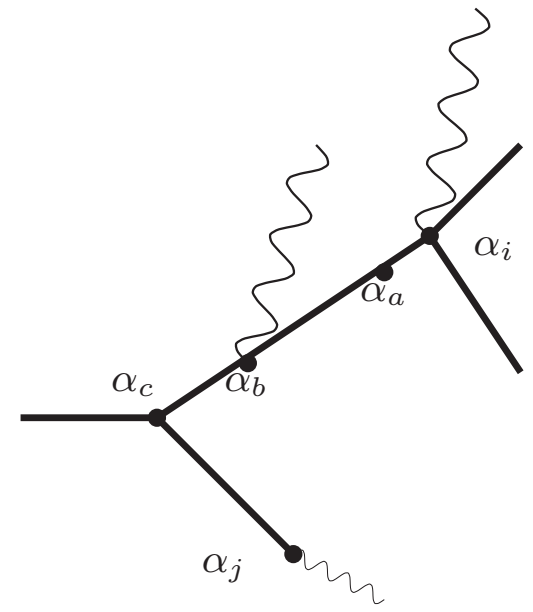

FIG. 2. A tree of propagators connecting vertices and external lines represented by wiggly lines; the dots are associated with $\delta_{\alpha}=(0,1,-1)$.

coordinates of two external lines,

$$
x_{i}-x_{j}=x_{i}^{\prime}+\rho_{i} \bar{x}_{l_{i}}-x_{j}^{\prime}-\rho_{j} \bar{x}_{l_{j}}=\sum_{\alpha}^{*} \delta_{\alpha},
$$

where the sum is over the vertices in the path of the tree connecting $i$ and $j$, and $\delta_{\alpha}=(0,1,-1)$ is associated with the line connected to the vertex $\alpha$. When $U=0$, then necessarily $l_{j}=l_{j}$. It is natural to distinguish among the terms contributing to the effective potential between resonant terms and nonresonant terms. The first are the contributions in (20) in which the coordinate $x^{\prime}$ of the external fields is equal, $\psi_{\rho_{1} ; x_{0,1}, x_{1}^{\prime}, l_{1}}^{\varepsilon_{1}(\leqslant h)} \cdots \psi_{\rho_{m} ; x_{0, m}, x_{1}^{\prime}, l_{m}}^{\varepsilon_{m}(\leqslant h)}$, that is, for any $i . j$,

$$
x_{i}^{\prime}=x_{j}^{\prime} .
$$

The nonresonant terms are the ones such that, for some $i, j$, $x_{i}^{\prime} \neq x_{j}^{\prime}$ so that from (26) and the second Melnikov condition (25)

$$
\begin{aligned}
2 \gamma^{h} & \geqslant\left\|\left(\omega x_{i}^{\prime}\right)\right\|+\left\|\left(\omega x_{j}^{\prime}\right)\right\| \geqslant\left\|\omega\left(x_{i}^{\prime}-x_{j}^{\prime}\right)\right\| \\
& =\left\|\omega\left(\rho_{i} \bar{x}_{l_{i}}-\rho_{j} \bar{x}_{l_{j}}\right)+\omega \sum_{\alpha}^{*} \delta_{\alpha}\right\| \geqslant \frac{C_{0}}{\left|\sum_{\alpha}^{*} \delta_{\alpha}\right|^{\tau^{\prime}}}
\end{aligned}
$$

so that $\left|\sum_{\alpha}^{*} \delta_{\alpha}\right| \geqslant \widetilde{C} \gamma^{-h / \tau}$.

One can then use the high power or $J, U$ to get a gain factor, making irrelevant the nonresonant contributions to the effective potential. Writing $\varepsilon=\max (|J|,|U|)$ and $\varepsilon=$ $\prod_{h=-\infty}^{0} \varepsilon^{2^{h-1}}$, we can associate a factor $\varepsilon^{2^{h_{v}-1}}$ for each end point enclosed in the cluster $v$; as $\left|\sum_{\alpha}^{*} \delta_{\alpha}\right|$ is surely smaller than the number of vertices in the cluster $v$, and choosing $\gamma^{\frac{1}{\tau}} / 2>1$, we can associate to each nonresonant contribution a factor $\varepsilon^{2^{h-1}\left|\sum_{\alpha}^{*} \delta_{\alpha}\right|} \leqslant \varepsilon^{C 2^{h} \gamma^{-h / \tau}} \leqslant \gamma^{4 h}$ for $\varepsilon$ small; therefore,

$$
\varepsilon^{\frac{n}{2}} \leqslant \prod_{v} \varepsilon^{C 2^{h_{v}} \gamma^{-h_{v} / \tau} S_{v}^{N R}} \leqslant \prod_{v} \gamma^{4 h_{v} S_{v}^{N R}},
$$

where $S_{v}^{N R}$ is the number of nonresonant clusters in $v$; this means that to each nonresonant term is associated at least a factor $\gamma^{4 h_{v}}$, which is sufficient to make its scaling dimension negative.
It remains to prove that (24) and (25) are true in a large relative measure set of values, that is, if $\left|J_{\perp}\right| \leqslant \varepsilon_{0}$, in a set whose complement has measure $O\left(C_{L} \varepsilon_{0}^{1+\alpha}\right), \alpha \geqslant 0$, and $C_{L}$ is an $L$-dependent constant. Indeed if (24) is true, then if $\cos l \neq 0$,

$$
\begin{aligned}
C_{0}|x|^{-\tau} & \leqslant\|\omega x \pm 2 \bar{x}\| \\
& \leqslant\left\|\omega x \pm 2\left(\bar{x}+a J_{\perp} \cos l\right)\right\|+2\left|a J_{\perp} \cos l\right| \\
& \leqslant C_{0}|x|^{-\tau^{\prime}}+C\left|\varepsilon_{0} \cos l a\right|
\end{aligned}
$$

so that if $\tau^{\prime}>\tau+1, C_{0} / 2|x|^{-\tau} \leqslant C_{0}|x|^{-\tau}\left(1-|x|^{\tau-\tau^{\prime}}\right) \leqslant$ $C\left|a \varepsilon_{0} \cos l\right|$ for $|x| \geqslant 2$, and $|x| \geqslant\left(2 C \varepsilon_{0} \cos l a \mid / C_{0}\right)^{\frac{-1}{\tau}}=\mathcal{N}_{0}$. The set $I$ of $J_{\perp}$ not verifying (24) is defined by the condition, for $-1 \leqslant s \leqslant 1$,

$$
f(s)=\omega x \pm 2\left[\bar{x}+J_{\perp}(s) \cos l a\right]=s C_{0}|x|^{-\tau^{\prime}}
$$

and $\frac{\partial f}{\partial s}=\frac{\partial f}{\partial J_{\perp}} \frac{\partial J_{\perp}}{\partial s}=C_{0}|x|^{-\tau^{\prime}}$ so that the measure of the region in which $(24)$ is not true is

$$
\begin{aligned}
\int_{I} d J_{\perp} & =\sum_{l} \sum_{n \geqslant \mathcal{N}_{0}} \int_{-1}^{1}\left|\frac{d J_{\perp}}{d s}\right| d s \\
& \leqslant \sum_{l, \cos l \neq 0} \frac{C}{\cos l} \sum_{x \geqslant \mathcal{N}_{0}}|x|^{-\tau^{\prime}} \leqslant C_{L}\left|\varepsilon_{0}\right|^{\frac{\tau^{\prime}-1}{\tau}}
\end{aligned}
$$

and choosing $\frac{\tau^{\prime}-1}{\tau}>1$, that is, $\tau^{\prime}>\tau+1$, we have that for $\left|J_{\perp}\right| \leqslant \varepsilon_{0}$ the relative measure of the excluded $J_{\perp}$ is $O\left(C_{L} \varepsilon_{0}^{\tau^{\prime}-\tau-1}\right)$, hence it is vanishing if $\varepsilon_{0} \rightarrow 0$. A similar procedure can be repeated for the second Melnikov condition; if $\cos l_{i} \pm \cos l_{j} \neq 0$, then

$$
\begin{aligned}
C_{0}|x|^{-\tau} \leqslant & || \omega x \pm\left(\bar{x}+a \cos l_{i} J_{\perp}\right) \pm\left(\bar{x}+a \cos l_{j} J_{\perp}\right) \| \\
& +\left|J_{\perp} a\left(\cos l_{i} \pm \cos l_{j}\right)\right| \\
\leqslant & C_{0}|x|^{-\tau^{\prime}}+C\left|\varepsilon_{0}\left(\cos l_{i} \pm \cos l_{j}\right) a\right|
\end{aligned}
$$

from which $|x| \geqslant\left[2 C \varepsilon_{0}\left|\left(\cos l_{i} \pm \cos l_{j}\right) a\right| / C_{0}\right]^{\frac{-1}{\tau}}$; one then proceeds as above with $\left|\cos l_{i} \pm \cos l_{j}\right|$ replacing $\left|\cos l_{i}\right|$.

\section{THE RESONANT TERMS}

We have seen in the preceding section that the nonresonant terms are irrelevant. We must then construct a renormalized expansion for the two-point function, extracting, at each RG iteration, the marginal and relevant part of the resonant terms. In this way, the two-point function is written as an expansion in a set of running coupling constants, which is convergent if such constants remain small at each scale; convergence at the end implies localization in the ground state at a nonperturbative level, as it means that the interacting theory is analytically close to the noninteracting one, which is localized.

We focus now on some properties of the resonant terms. Note that $x_{i}-x_{j}=x_{i}^{\prime}-x_{j}^{\prime}+\rho_{i} \bar{x}_{l_{i}}-\rho_{j} \bar{x}_{l_{j}} \in \mathbb{Z}$ so that in the resonances $\rho_{i} \bar{x}_{l_{i}}-\rho_{j} \bar{x}_{l_{j}} \in \mathbb{Z}$. This says that, up to a zero measure set of $J_{\perp}, \rho_{i} \bar{x}_{l_{i}}-\rho_{j} \bar{x}_{l_{j}}=0$ as $\left(\cos l_{i}-\cos l_{j}\right) a J_{\perp}$ or $2 \bar{x}+\left(\cos l_{i}+\cos l_{j}\right) a J_{\perp}$ can be a nonvanishing integer only in a zero measure set (by the diophantine condition, $2 \bar{x}$ cannot be integer). In addition in a resonant terms, all the fields necessarily have the same $\rho$,

$$
\rho_{i}=\rho_{j}
$$


as if $\rho_{i}=-\rho_{j}$ one gets $2 \bar{x}+\left(\cos l_{i}+\cos l_{j}\right) a J_{\perp}=0$, which cannot be vanishing for small $J_{\perp}$. Finally in the resonances the condition $\left(\cos l_{i}-\cos l_{j}\right) a J_{\perp} \neq 0$ cannot be verified unless $\cos l_{i}=\cos l_{j}$. The above properties imply that the resonances with a number of fields $\geqslant 4$ have the following structure:

$$
\prod_{i} \psi_{\rho ; x^{\prime}, x_{0, i}, l_{i}}^{\varepsilon_{i}}, \quad \cos l_{j}=\cos l_{j} .
$$

If $L=2$, that is, the array is only composed of two chains, then $l=(0, \pi), \bar{x}_{1}=\bar{x}+J_{\perp} / 2$, and $\bar{x}_{1}=\bar{x}-J_{\perp} / 2$ so that the resonant terms have the same $\rho, l$ index; this has the effect that the monomials with $\geqslant 4$ fields and the same coordinates are vanishing. In resonances for which the number of fields is greater than two, there are at least two couples of the form $\psi_{\rho ; x^{\prime}, x_{0,1}, l}^{\varepsilon} \psi_{\rho ; x^{\prime}, x_{0,2}, l}^{\varepsilon}$, which can be rewritten as

$$
\psi_{\rho ; x^{\prime}, x_{0,1}, l}^{\varepsilon} \psi_{\rho ; x^{\prime}, x_{0,2}, l}^{\varepsilon}=\psi_{\rho ; x^{\prime}, x_{0,1}, l}^{\varepsilon}\left(\psi_{\rho ; x^{\prime}, x_{0,2}, l}^{\varepsilon}-\psi_{\rho ; x^{\prime}, x_{0,1}, l}^{\varepsilon}\right)
$$

and

$$
\psi_{\rho ; x^{\prime}, x_{0,2}, l}^{\varepsilon}-\psi_{\rho ; x^{\prime}, x_{0,1}, l}^{\varepsilon}=\left(x_{0,2}-x_{0,1}\right) \int_{0}^{1} d t \partial \psi_{\rho ; x^{\prime}, x_{0}(t), l}^{\varepsilon}
$$

with $x_{0}(t)=x_{0,1}+t\left(x_{0,2}-x_{0,1}\right)$. The derivative produces an extra $\gamma^{h_{v^{\prime}}}$ if $v^{\prime}$ is the cluster enclosing $v$, and the factor $\left(x_{0,2}-\right.$ $\left.x_{0,1}\right)$ produces an extra $\gamma^{-h_{v}}$; as there are at least two such monomials, one gets at least a factor $\gamma^{2\left(h_{v^{\prime}}-h_{v}\right)}$. Remembering that the scaling dimension is $D=1$, this means that all the resonances with more than two fields are irrelevant if $L=2$.

If $L \geqslant 3$, the situation is different; there are a couple of indices $l, l^{\prime}$ such that $x_{l}=x_{l^{\prime}}$; quartic terms involving such indices and the same $x_{0, i}$ are not vanishing so that there are quartic relevant terms. For instance, in the three-chain problem $L=3$ one has $l=2 \pi / 3,4 \pi / 3,6 \pi / 3$ and $\bar{x}_{1}=\bar{x}-J_{\perp} / 2, \bar{x}_{1}=$ $\bar{x}-J_{\perp} / 2, \bar{x}_{3}=\bar{x}$; the local part of the quartic terms (the part with identical coordinates) $\psi_{\rho ; \mathbf{x}^{\prime}, 1}^{+} \psi_{\rho ; \mathbf{x}^{\prime}, 1}^{-} \psi_{\rho ; \mathbf{x}^{\prime}, 2}^{+} \psi_{\rho ; \mathbf{x}^{\prime}, 2}^{-}$is nonvanishing; the quartic terms are indeed relevant, while resonant terms with a number greater than 6 are irrelevant. The number of couples $i, j$ with $\cos l_{i}=\cos l_{j}$, and the corresponding quartic terms, increases with $L$; for instance, for $L=8$ one has $l=\pi / 4, \pi / 2,3 \pi / 4, \pi, 5 \pi / 4,3 \pi / 2,7 \pi / 4,2 \pi$ with $\cos l=$ $\sqrt{2} / 2,0,-\sqrt{2} / 2,-1,-\sqrt{2} / 2,0, \sqrt{2} / 2,1$, so that the nonvanishing local quartic terms are $\psi_{\rho ; \mathbf{x}^{\prime}, 1}^{+} \psi_{\rho ; \mathbf{x}^{\prime}, 1}^{-} \psi_{\rho ; \mathbf{x}^{\prime}, 7}^{+} \psi_{\rho ; \mathbf{x}^{\prime}, 7}^{-}$, $\psi_{\rho ; \mathbf{x}^{\prime}, 2}^{+} \psi_{\rho ; \mathbf{x}^{\prime}, \rho, 2}^{-} \psi_{\rho ; \mathbf{x}^{\prime}, 6}^{+} \psi_{\rho ; \mathbf{x}^{\prime}, 6}^{-}, \quad \psi_{\rho ; \mathbf{x}^{\prime}, 3}^{+} \psi_{\rho ; \mathbf{x}^{\prime}, 3}^{-} \psi_{\rho ; \mathbf{x}^{\prime}, \rho, 5}^{+} \psi_{\rho ; \mathbf{x}^{\prime}, 5}^{-}$. As there are at most a couple of fields with the same $\bar{x}_{l}$ and different $l$, the terms with a number $\geqslant 6$ of fields are irrelevant, as there are at least four fields with the same $l$.

To get a convergent expansion, one has to extract the relevant part from the resonant terms. If $V_{\text {res }}^{h}=\sum_{m} V_{m \text {,res }}^{h}$, where $V_{m}^{h}$ are the monomials with $m$ fields, then we define a localization operation $V_{2}^{h}=\mathcal{L} V_{2}^{h}+\mathcal{R} V_{2}^{h}$ with $\mathcal{R}=1-\mathcal{L}$, and $\mathcal{L}$ acts on the kernels of $V_{2}^{h}$ in the following way:

$$
\mathcal{L} \widehat{W}_{2}^{h}\left(k_{0}, x^{\prime}\right)=\widehat{W}_{2}^{h}(0,0)+k_{0} \partial_{0} \widehat{W}_{2}^{h}(0,0)+\left(\omega x^{\prime}\right) \widetilde{\partial} W_{2}^{h}(0,0),
$$

where $\widetilde{\partial} \widehat{W}_{2}^{h}\left(k_{0}, x^{\prime}\right)=\frac{\widetilde{W}_{2}^{h}\left(k_{0}, x^{\prime}\right)-\widetilde{W}_{2}^{h}\left(k_{0}, 0\right)}{\left(\omega x^{\prime}\right)}$. The action of $\mathcal{R}=1-$ $\mathcal{L}$ produces a gain $\gamma^{2\left(h_{v^{\prime}}-h_{v}\right)}$, using also that $\left(\omega x^{\prime}\right)^{2} \sim \gamma^{2 h_{v^{\prime}}}$, if $v^{\prime}$ is the smallest cluster enclosing $v$ for the compact support properties of the lines external to the cluster $v$, while $\widetilde{\partial}^{2} \widehat{W}_{2}^{h}\left(k_{0}, x^{\prime}\right)$ has an extra $\gamma^{-2 h_{v}} ; \mathcal{R} V_{2}^{h}$ is therefore irrelevant. The local part of the effective potential then has the form

$$
\begin{aligned}
\mathcal{L} \mathcal{V}_{\mathrm{res}, 2}^{h}= & \sum_{x} \frac{1}{L} \sum_{\rho, l} \int d x_{0}\left[v_{h, l} \gamma^{h} \psi_{\rho ; \mathbf{x}^{\prime}, l}^{+} \psi_{\rho ; \mathbf{x}^{\prime}, l}^{+}\right. \\
& \left.+z_{h, l, \rho} \psi_{\rho ; \mathbf{x}^{\prime}, l}^{+} \partial_{0} \psi_{\rho ; \mathbf{x}^{\prime}, l}^{+}+\alpha_{h, l, \rho}\left(\omega x^{\prime}\right) \psi_{\rho ; \mathbf{x}^{\prime}, l}^{+} \psi_{\rho ; \mathbf{x}, l}^{+}\right] .
\end{aligned}
$$

Regarding terms with a number of fields $\geqslant 6, \mathcal{L} V_{\text {res }, m}^{h}=0$ for $m \geqslant 6$, as the local part and its first derivative are vanishing. Finally, if $L=1,2$, then

$$
\mathcal{L} V_{\text {res }, 4}^{h}=0
$$

while for $L \geqslant 3$ then

$$
\begin{aligned}
\mathcal{L} V_{\mathrm{res}, 4}= & G+\frac{1}{L^{3}} \sum_{i, j ; \bar{x}_{i}=\bar{x}_{j}} \sum_{\rho} \lambda_{h, i, j, \rho} \gamma^{h} \\
& \times \int d \mathbf{x} \psi_{\rho ; \mathbf{x}^{\prime}, i}^{+} \psi_{\rho ; \mathbf{x}^{\prime}, i}^{-} \psi_{\rho ; \mathbf{x}^{\prime}, j}^{+} \psi_{\rho ; \mathbf{x}^{\prime}, j}^{-},
\end{aligned}
$$

where marginal terms are included in $G$, that is, quartic local terms with at least a field $\partial \psi$ (the corresponding coupling is called $\left.\widetilde{\lambda}_{h, i, j, \rho}\right)$ and the sum $\sum_{i, j ; \bar{x}_{i}=\bar{x}_{j}}$ is over the fields with the same $\bar{x}_{l}$.

\section{CONVERGENCE OF THE RENORMALIZED EXPANSION}

The integration is done separating at each integration step the relevant and the irrelevant parts of the effective integration, writing

$$
\int P\left(d \psi^{\leqslant h}\right) e^{\mathcal{L} V^{(h)}\left(\psi^{\leqslant h}\right)+\mathcal{R} V^{(h)}\left(\psi^{\leqslant h}\right)}
$$

with $\mathcal{R}=1-\mathcal{L}$, and $\mathcal{L}$ is the localization operator defined above; this allows us to get an expansion in terms of running coupling constants $\vec{v}_{h}=\left(\lambda_{h, l, l^{\prime}, \rho}, \widetilde{\lambda}_{h, l, l^{\prime}, \rho}, v_{h, l}, \alpha_{h, l}, z_{h, l}\right)$. If $v_{0}$ is the largest cluster, $v$ are the clusters (without vertices), $\bar{v}$ are the vertices, $R$ or $N R$ are the resonant clusters or vertices, and $v^{\prime}$ is the first cluster enclosing $v$, then

$$
\prod_{v} \gamma^{-h_{v} S_{v}}=\prod_{v \neq v_{0}} \gamma^{-h_{v^{\prime}}} \prod_{\bar{v}} \gamma^{-h_{\bar{v}^{\prime}}}
$$

and $\prod_{v} \gamma^{h_{v}}=\gamma^{h_{v_{0}}} \prod_{v \neq v_{0}} \gamma^{h_{v}}$ so that (22) can be rewritten as

$$
\varepsilon^{n} \gamma^{h_{v_{0}}} \prod_{v \neq v_{0}} \gamma^{-\left(h_{v^{\prime}}-h_{v}\right)} \prod_{\bar{v}} \gamma^{-h_{\bar{v}^{\prime}}}
$$

Using (29), we get that the kernel $W_{m}^{(h)}$ in the renormalized expansion is bounded, if $\left|\bar{v}_{h}\right| \leqslant \varepsilon$, by

$$
\begin{gathered}
{\left[\varepsilon^{n / 2} \prod_{v} \gamma^{-h_{v}\left(S_{v}-1\right)}\right]\left[\prod_{v \in R} \gamma^{2\left(h_{v^{\prime}}-h_{v}\right)}\right]} \\
\times\left[\prod_{\bar{v} \in R} \gamma^{h_{\bar{v}^{\prime}}}\right]\left[\prod_{v} \gamma^{4 S_{v}^{N R} h_{v}}\right],
\end{gathered}
$$

where the factor $\left[\prod_{v \in R} \gamma^{2\left(h_{v^{\prime}}-h_{v}\right)}\right]$ is, as explained in the previous section, due to the action of $\mathcal{R}$ or to (35) and (36). 
Therefore, (43) can be written as

$$
C^{n} \varepsilon^{n / 2} \gamma^{h_{v_{0}}}\left[\prod_{v} \gamma^{\left(h_{v^{\prime}}-h_{v}\right)}\right]\left[\prod_{\bar{v} \in N R} \gamma^{h_{\bar{v}^{\prime}}}\right] .
$$

As $h_{v^{\prime}}-h_{v} \leqslant 0$, it is possible over the scales $h_{v}$ to obtain a bound followed by convergence provided that $\varepsilon$ is not too large. Note that the above bound is valid for the sum of all Feynman graphs of order $n$ by using determinant bounds for fermionic expectations; see [28] for details. The renormalized expansion has a finite radius of convergence in terms of the running coupling constants; if they remain, for any $h$, then localization is found inside the convergence radius. We must then analyze the flow of the effective couplings, and the result is dramatically different in the $L=2$ and $L \geqslant 3$ cases.

In the $L=2$ case, there are no quartic terms in the effective potential, that is, $\lambda_{h}=\widetilde{\lambda}_{h}=0$; the only effective couplings are quadratic, and the flow equations are

$$
v_{h-1, l}=\gamma v_{h}+\beta_{h, l}^{v}, \quad \alpha_{h-1, l}=\alpha_{h}+\beta_{h, l}^{\alpha},
$$

and $z_{h-1}=z_{h}+\beta_{h, l}^{z}$, where $\beta_{h}^{v}, \beta_{h}^{\alpha}, \beta_{h}^{z}$ are the beta functions; they are given by a sum of terms with at least one irrelevant term, as terms containing only marginal terms (quadratic in the fields) are chain graphs giving a vanishing contribution to be a $\beta$ function via the compact support properties of the propagator. Therefore, by (44), the $\beta$ function is asymptotically vanishing, $\beta_{h}^{v}, \beta_{h}^{\alpha}, \beta_{h}^{z}=O\left(\gamma^{h}\right) . v_{h, l}$ is a relevant coupling, but its flow can be controlled by choosing properly the counterterms $v_{l}$; indeed, we can write $v_{h-1, l}=\gamma^{-h}\left(v_{l}+\sum_{k=h}^{0} \gamma^{k} \beta_{k, l}^{v}\right)$, and choosing $v_{l}=-\sum_{k=-\infty}^{0} \gamma^{k} \beta_{k, l}^{v}$ we obtain $v_{h, l}=O\left(\gamma^{h} \varepsilon\right)$. Moreover, $\alpha_{h-1, l}=\sum_{k=h}^{0} \beta_{h, l}^{\alpha}=O(\varepsilon)$ and similarly $\alpha_{h-1, l}=$ $O(\varepsilon)$. Therefore, if $J, U$ are sufficiently small, we have that the running coupling constants are small and the series are convergent. Similarly, if $L \geqslant 3$ and $U=0$ there are only quadratic couplings, and we can proceed in the same way.
In the case $L \geqslant 3$, however, there are quartic relevant and marginal couplings, that is,

$$
\lambda_{h-1, l, l^{\prime}, \rho}=\gamma \lambda_{h, l, l^{\prime}, \rho}+\beta_{h, l, l^{\prime}, \rho}^{\lambda} .
$$

Convergence is achieved at finite temperatures, that is, for $\gamma^{-h_{\beta}} U$ or $\beta U$ of order 1 , and at lower temperatures one expects generically an unbounded flow.

An estimate for the two-point function follows easily from the expansion for the effective potential; if the external coordinates are $x$ and $y$, then there are at least $|x-y| \varepsilon$ factors, and this implies exponential decay in the direction of the chains. By the first Melnikov condition, the smallest scale of the contribution at order $n$ verifies

$$
\gamma^{-\bar{h}} \leqslant C(1+\min \{|x|,|y|\})^{\tau}\left(1+\frac{n}{1+\min \{|x|,|y|\}}\right)^{\tau}
$$

from which follows Eq. (13).

\section{CONCLUSIONS}

We have considered an array of interacting chains with quasirandom disorder. The RG analysis is in agreement with cold-atoms experiments [24,25], in which it is found that localization is present in the single-chain case while it is absent when several chains are considered. In the first case, numbertheoretical properties, combined with cancellations due to the Pauli principle, ensure that all the effective interactions are irrelevant, even if dimensionally relevant. On the contrary, in the second case there are nonvanishing relevant interactions, whose number increases with the number of chains; as usual, the presence of diverging directions in the RG flow is expected to signal an instability of the system. In addition, we have shown that localization in the ground state is present with two chains if the fermions are spinless, and in the presence of interaction a prediction in principle is accessible with experimental verification.
[1] P. W. Anderson, Absence of diffusion in certain random lattices, Phys. Rev. 109, 1492 (1958).

[2] R. Nandkshore and D. Huse, Many body localization and thermalization in quantum statistical mechanics, Annu. Rev. Condens. Matter Phys. 6, 15 (2015).

[3] C. Gogolin and J. Eisert, Equilibration, thermalisation, and the emergence of statistical mechanics in closed quantum systems, Rep. Prog. Phys. 79, 056001 (2016).

[4] S. Goldstein, D. A. Huse, J. L. Lebowitz, and R. Tumulka, Thermal Equilibrium of a Macroscopic Quantum System in a Pure State, Phys. Rev. Lett. 115, 100402 (2015).

[5] L. Fleishmann and P. W. Anderson, Interactions and the Anderson transition, Phys. Rev B 21, 2366 (1980).

[6] I. V. Gornyi, A. D. Mirlin, and D. G. Polyakov, Interacting Electrons in Disordered Wires, Phys. Rev. Lett. 95, 206603 (2005).

[7] D. M. Basko, I. Alteiner, and B. L. Altshuler, Metal-insulator transition in a weakly interacting many-electron system with localized single-particle states, Ann. Phys. (NY) 321, 1126 (2006).
[8] M. Serbyn, Z. Papic, and A. Abanin, Local Conservation Laws and the Structure of the Many-Body Localized States, Phys. Rev. Lett. 111, 127201 (2013).

[9] V. Ros, M. Mueller, and A. Scardicchio, Integrals of motion in the many-body localized phase, Nucl. Phys. B 891, 420 (2015).

[10] J. Imbrie, On many-body localization for quantum spin chains, J. Stat. Phys. 163, 998 (2016).

[11] J. Imbrie, Diagonalization and Many-Body Localization for a Disordered Quantum Spin Chain, Phys. Rev. Lett. 117, 027201 (2016).

[12] V. Oganesyan and D. A. Huse, Localization of interacting fermions at high temperature, Phys. Rev. B 75, 155111 (2007).

[13] A. Pal and D. A. Huse, Many-body localization phase transition, Phys. Rev. B 82, 174411 (2010).

[14] M. Znidariic, T. Prosen, and P. Prelovsek, Many-body localization in the Heisenberg XXZ magnet in a random field, Phys. Rev. B 77, 064426 (2008).

[15] S. Aubry and G. André, Analyticity breaking and Anderson localization in incommensurate lattices, Ann. Isr. Phys. Soc. 3, 133 (1980). 
[16] J. Froehlich, T. Spencer, and T. Wittwer, Localization for a class of one-dimensional quasi-periodic Schrödinger operators, Commun. Math. Phys. 132, 5 (1990).

[17] S. Jitomirskaya, Metal-insulator transition for the almost Mathieu operator, Ann. Math. 150, 1159 (1999).

[18] V. Mastropietro, Localization in the ground state of an interacting quasi-periodic fermionic chain, Commun. Math. Phys. 342, 217 (2016); Localization of Interacting Fermions in the Aubry-André Model, Phys. Rev. Lett. 115, 180401 (2015); Localization in interacting fermionic chains with quasi-random disorder, Commun. Math. Phys. 351, 283 (2017).

[19] S. Iyer, V. Oganesyan, G. Refael, and D. A. Huse, Many-body localization in a quasiperiodic system, Phys. Rev. B 87, 134202 (2013).

[20] F. Iemini, A. Russomanno, D. Rossini, A. Scardicchio, and R. Fazio, Signatures of many-body localisation in the dynamics of two-sites entanglement, Phys. Rev. B 94, 214206 (2016).

[21] S. Bera, H. Schomerus, F. Heidrich-Meisner, and J. H. Bardarson, Many-Body Localization Characterized from a OneParticle Perspective, Phys. Rev. Lett. 115, 046603 (2015).
[22] P. Naldesi, E. Ercolessi, and T. Roscilde, Detecting a many-body mobility edge with quantum quenches, SciPost Phys. 1, 010 (2016).

[23] P. Prelovsek, O. S. Barisich, and M. Znidaric, Absence of full many-body localization in disordered Hubbard chain, Phys. Rev. B 94, 241104 (2016).

[24] M. Schreiber, S. Hodgman. P. Bordia, H. P. Lschen, M. H. Fischer, R. Vosk, E. Altman, U. Schneider, and I. Bloch, Observation of many-body localization of interacting fermions in a quasi random optical lattice, Science 349, 842 (2015).

[25] P. Bordia, H. Lschen, S. Hodgman, M. Schreiber, I. Bloch, and U. Schneider, Coupling Identical 1D Many-Body Localized Systems, Phys. Rev. Lett. 116, 140401 (2016).

[26] L. H. Eliasson, Perturbations of Linear Quasi-periodic System. Dynamical Systems and Small Divisors (Cetraro, 1998), Lecture Notes in Math Vol. 1784 (Springer, Berlin, 2002), pp. 1-60.

[27] D. L. Shepelyansky, Two interacting particles in the Harper model, Phys. Rev. B 54, 14896 (1996).

[28] V. Mastropietro, Nonperturbative Renormalization (World Scientific, Singapore, 2008). 